\section{Stevens-Johnson syndrome/ erythema multiforme major and Chlamydia pneumoniae infection in young patients}

\author{
Ana Filipa Duarte, ${ }^{1}$ Maria João Cruz, ${ }^{2}$ \\ Elisabete Moreira, ${ }^{2}$ Teresa Baudrier, \\ Alberto Mota, ${ }^{1}$ Filomena Azevedo ${ }^{2}$ \\ 'Hospital São João, EPE and Faculty of \\ Medicine, Porto, Portugal; \\ ${ }^{2}$ Hospital São João, EPE, Porto, Portugal
}

\section{Abstract}

Erythema multiforme major (EMM) is an acute, self-limited mucocutaneous disease characterized by the abrupt onset of symmetrical fixed red papules evolving to target lesions. It is triggered mainly by infections, such as herpes simplex virus (HSV) and Mycoplasma pneumoniae, or drugs. In instances of extensive skin lesions with "giant" targets, prominent involvement of several mucous sites and fever, it may be difficult to distinguish from Stevens-Johnson syndrome (SJS), a rarer, lifethreatening reaction which is mainly druginduced. We report a 7-year old boy with SJS and Chlamydia pneumoniae infection and 3 patients with EM and co-infection with Chlamydia pneumoniae: a 3 -year old girl and a 29-year old man developed EMM lesions associated to Mycoplasma pneumoniae and Chlamydia pneumonia and a 20-year old woman with EMM associated to herpes simplex type 2 and Chlamydia pneumoniae infection. None of the 4 patients had history of drug intake in the last two months.

Chlamydia pneumoniae is an intracellular bacteria responsible for respiratory infections. Despite the fact that its role in SJS/EMM has been rarely reported, our cases suggest that it may cause SJS and trigger EM when co-infecting a patient, either with Mycoplasma pneumoniae or herpes simplex. We conclude that infection by Chlamydia pneumoniae should be suspected and ruled out in every patient with SJS/EMM, especially in those with signs of respiratory infection.

\section{Introduction}

Erythema multiforme major (EMM) is an acute, self-limiting mucocutaneous disease characterized by the abrupt onset of symmetrical fixed red papules, located primarily on the extremities, some evolving to target lesions. ${ }^{1}$ The incidence of EM has been estimated to vary between $0.01 \%$ and $1 \% .{ }^{2}$ It is triggered mainly by infections, such as herpes simplex virus (HSV) and Mycoplasma pneumoniae, or drugs. In instances of extensive skin lesions with "giant" atypical targets, centripetal distibution, prominent involvement of several mucous sites and fever, it may be difficult to distinguish from Stevens-Johnson syndrome (SJS), a rarer (1-6 cases per million) and lifethreatening reaction which is mainly druginduced. ${ }^{3}$

\section{Case Reports}

We report a 7-year-old boy with a rapidly progressive macular-papular exanthema evolving to atypical target lesions involving less than $10 \%$ of the body surface, oral mucosa erosions, conjunctivitis and fever $\left(38^{\circ} \mathrm{C}\right)$ in less than 24 hours (Patient \#1 - Figure 1). There was no history of drug intake in the past two months. Nicolsky sign was positive. Pulmonary auscultation was normal, but the X-ray revealed a bilateral interstitial infiltrate, compatible with atypical pneumonia. Skin biopsy specimen taken from the border of a lesion on the back, showed dermo-epidermal detachment, apoptotic keratinocytes and inflammatory cell infiltrate, compatible with SJS (Figure 2).

Serum IgG for $C$. pneumoniae was negative and $\operatorname{IgM}$ was $3.4 \mathrm{U} / \mathrm{mL}$ (normal $<0.5 \mathrm{U} / \mathrm{mL}$ ) on admittance but turned frankly positive (36.7 $\mathrm{U} / \mathrm{mL}$ ) four weeks later. The remaining investigation showed mild leukocytosis with neutrophilia and an elevated reactive $\mathrm{C}$ protein. Blood and urine cultures were negative. Serologies to Herpes simplex, M. pneumoniae, Parvovirus B19, Varicela zoster virus, Human Immunodeficiency Virus (HIV), Hepatitis B and $\mathrm{C}$, Venereal Disease Research Laboratory (VDRL), T. pallidum Human Agglutination (TPHA) and urine Legionella pneumoniae antigen, were all negative.

Oral clarithromycin $500 \mathrm{mg}$ twice a day for ten days and topical bethametasone twice a day for five days and once a day for a further five days were prescribed with complete clinical resolution in ten days.

In addition 3 other cases of $C$. pneumoniae were demonstrated as co-infection in patients with EMM.

Patient \#2, a 3-year old girl (Figure 3 ) developed a confluent maculopapular exanthema evolving to target lesions, localized to the face, chest and limbs, associated with fever and conjunctival hyperemia. Serum IgM for both $M$. pneumoniae and $C$. pneumoniae was reactive by enzyme immunoassay and IgG was negative suggesting recent infection.

Patient \#3, a 29-year old man (Figure 4), presented with a maculopapular exanthema and target lesions, localized to the face, trunk and limbs, fever and conjunctival hyperemia.
Correspondence: Ana Filipa Duarte, Department of Dermatology and Venereology, Hospital São João, Alameda Professor Hernâni Monteiro 4200319 Porto, Portugal.

E-mail: duarte.af.t30@gmail.com

Key words: Stevens-Johnson syndrome, erythema multiforme major, Chlamydia pneumoniae.

Received for publication: 27 December 2009.

Revision received: 2 March 2010.

Accepted for publication: 3 March 2010.

This work is licensed under a Creative Commons Attribution 3.0 License (by-nc 3.0).

CCopyright A.F. Duarte et al., 2010

Licensee PAGEPress, Italy

Dermatology Reports 2010; 2:e6

doi:10.4081/dr.2010.e6

Serology suggested a recent infection for both $M$. pneumoniae and $C$. pneumoniae.

Patient \#4, a 20-year old woman presented with a generalized targetoid eruption, cheilitis and fever. Serological tests were positive for Herpes simplex type 2 IgM, C. pneumoniae IgM and Herpes simplex type 1 IgG.

Biopsy was performed on the 3 cases, showing interface spongiosis, individual necrotic keratinocytes and perivascular inflammatory cell infiltrate, supporting the diagnosis of erythema multiforme. Chest radiography was normal in the 3 cases. Complete blood count revealed mild leukocytosis with neutrophilia and an elevated reactive $\mathrm{C}$ protein. Polymerase chain reaction for Herpes simplex in the biopsy specimen was negative. The remaining investigation was irrelevant. None of the 3 patients had history of previous drug intake.

Patients \#2 and \#3 were treated with azithromycin $500 \mathrm{mg}$ per day for three days and topical bethametasone twice a day for five days and once a day for a further five days. Patient \#4 took clarithromycin $500 \mathrm{mg}$ twice a day for ten days and topical bethametasone twice a day for five days and once a day for a further five days. In the 3 cases, the eruption subsided gradually, with complete resolution in 2-4 weeks. A few months after convalescence, seroconversion was observed. IgM for Herpes simplex type 2 and $C$. pneumoniae became negative, but a positive IgG was detected in the case of patient \#4. In patients \#2 and \#3, IgM for $C$. pneumoniae and $M$. pneumoniae turned negative and IgG turned positive.

\section{Discussion}

C. pneumoniae is a human Gram-negative pathogen responsible for $5-20 \%$ of communityacquired pneumonias in adults and children. 


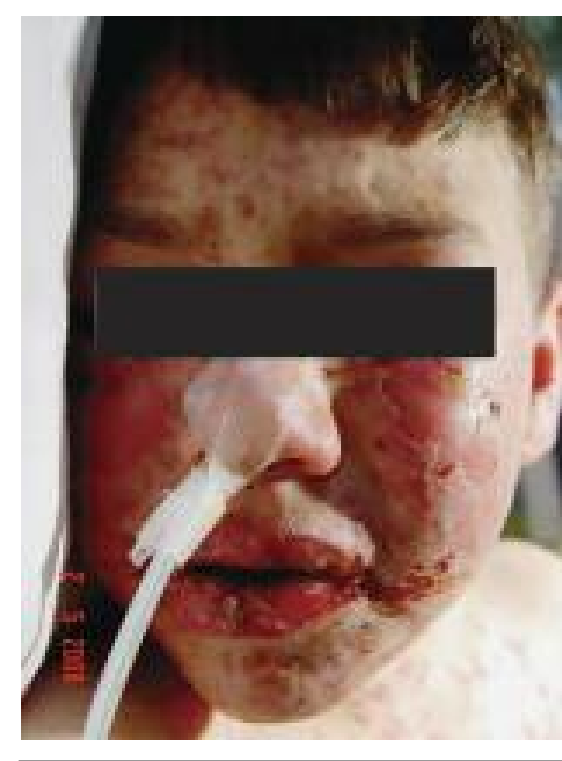

Figure 1. Patient \#1: atypical target lesions and erosions.

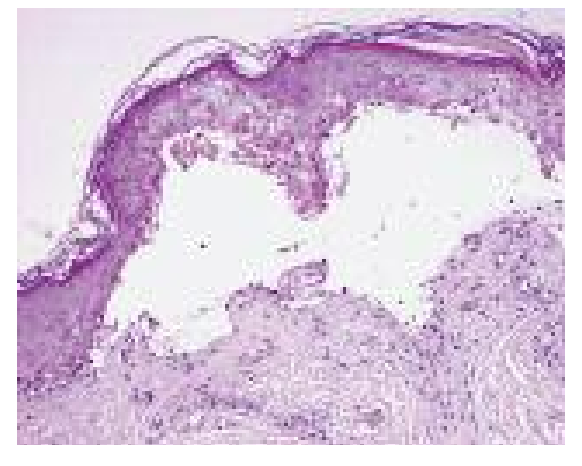

Figure 2. Patient \#1: H\&E (x40) - interface spongiosis, individual necrotic keratinocytes and perivascular inflammatory cell infiltrate.

It is a significant cause of both lower and upper acute respiratory illness, including atypical pneumonia, bronchitis, pharyngitis and sinusitis, but most cases are mild or asymptomatic. $^{5}$ The seroprevalence progressively increases with age, reaching $50 \%$ of antibodies against $C$. pneumoniae in the adult and exceeding $80 \%$ in the ederly. ${ }^{6}$

The ethiopathology of SJS and EMM is poorly understood, but some mechanisms, like immune-mediated vascular injury, cell-mediated immune response and autoimmune mechanisms are possible. ${ }^{7.8} \mathrm{EM}$ is often associated with HSV, type 1 in $50 \%$ of cases and less often type $2 .^{8,9}$ The second most reported agent is $M$. pneumoniae, occurring in about $5 \%$ of cases, but other agents may be implicated. ${ }^{8}$ In the cases of SJS, drugs are the most important etiologic factors.

The most commonly used method for the diagnosis of $C$. pneumoniae is serology, which

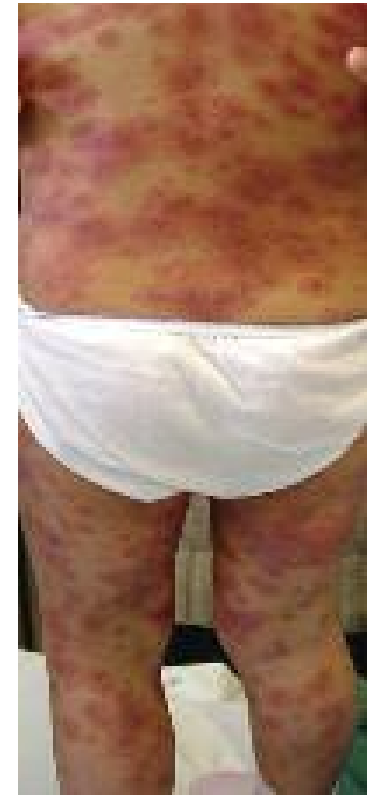

Figure 3. Patient \#2: confluent maculopapular exanthema evolving to target lesions.

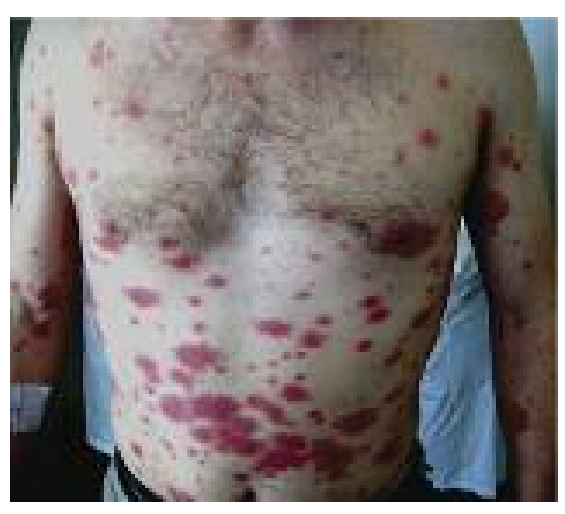

Figure 4. Patient \#3: atypical target lesions.

basically offers a retrospective study. In our 4 patients, a microimmunofluorescence test, which is considered as the "gold standard " of acute infections because of high sensitivity and specificity, was performed. ${ }^{10}$ For acute infection the patient should show a 4-fold increase in the IgG titre, an IgG titre of over 1:512 or a positive IgM titre. On prime infection, the IgM response usually appears at least three weeks after the onset of illness and the IgG response at 6-8 weeks. Because of the relatively long window for serological response in a primary infection, the antibody response may be missed if convalescent sera are obtained too soon. In fact, in some cases of acute infection, three months were needed for antibodies to be detected. Although PCR holds promise as a fast diagnostic tool, there are no standardized PCR or other nucleic acid amplification tests for detection of $C$. pneumoniae. ${ }^{4}$

Since no other predisposing factor was pres- ent, and serology confirmed acute infection, Patient \#1 had SJS due to $C$. pneumoniae infection. The other 3 patients had serologies suggesting that $C$. pneumoniae was at least a co-responsible factor for EMM.

Despite the fact that its role in this skin disease has been rarely reported, our cases support the view that a patient co-infection with $C$. pneumoniae and either with $M$. pneumoniae or Herpes simplex, seems to trigger EMM. It is possible that the presence of more than one infectious agent that includes $C$. pneumoniae may predispose patients to a more probable and/or more severe cutaneous reaction.

Besides SJS/EMM, C. pneumoniae is also associated with other skin reactions, including erythema nodosum, and hypersensitivity and Sweet syndromes. ${ }^{8}$

To the best of our knowledge, there has only been one report of 2 patients with $C$. pneumoniae and $M$. pneumoniae co-infection associated with EMM11, and one report of EMM associated to $C$. pneumoniae alone. ${ }^{8}$ No reports were found of EM associated to $C$. pneumoniae and Herpes simplex type 2 co-infection.

We believe that SJS/EMM associated with $C$. pneumoniae is underdiagnosed, because it has been rarely reported and physicians are not sufficiently aware to search for it. ${ }^{1,2,7,9}$ Patients presenting with SJS/EMM should be evaluated for active and latent HSV infection and appropriate serological testing for $M$. pneumoniae. ${ }^{9}$ However, other infectious causes should be considered. A normal chest X-ray does not exclude $M$. pneumoniae or $C$. pneumoniae infection, because the patient does not necessarily disclose pneumonia despite a relevant infection, besides the fact that atypical pneumonia without evident radiological image can be present. ${ }^{9}$ In the light of this, we think it is advisable to suspect those agents in patients with respiratory infection signs and SJS/EMM.

\section{References}

1. Léauté-Lambrèze $\mathrm{C}$, Lamireau $\mathrm{T}$, Chawki D, et al. Diagnosis, classification, and management of erythema multiforme and Stevens-Johnson syndrome. Arch Dis Child 2000;83:347-52.

2. Lam N-S, Yang Y-H, Wang L-C, et al. Clinical characteristics of childhood erythema multiforme, Stevens-Johnson syndrome and toxic epidermal necrolysis in Taiwanese children. J Microbiol Immunol Infect 2004;37:366-70.

3. Forman R, Koren G, Shear NH. Erythema multiforme, Stevens-Johnson syndrome and toxic epidermal necrolysis in children: a review of 10 years experience. Drugs Saf 2002;25:965

4. Hammerschlag MR. Chlamydia pneumoni- 
ae and the lung. Eur Respir J 2000;16: 1001-7.

5. Miyashita N. Chlamydia pneumoniae infections. Kekkaku 2006;81:581-8.

6. Hammerschlag MR. The intracellular life of chlamydiae. Semin Pediatr Infect Dis 2002;13:239-48.

7. Schalock PC, Dinulos JGH, Pace N, et al. Erythema multiforme due to Mycoplasma pneumoniae infection in two children. Pediatric Dermatology 2006;23:546-55.

8. Saada D, Velasco S, Vabres P, Guillet G. Érytheme polymorphe majeur et infection à Chlamydia pneumoniae. Ann Dermatol Venereol 2006;33:1001-4.

9. Schalock PC, Brennick JB, Dinulos JGH. Mycoplasma pneumoniae infection associated with bullous erythema multiforme. $\mathrm{J}$
Am Acad Dermatol 2005;52:705-6.

10. Wang S. The microimmunofluoresence test for Chlamydia pneumonia infection: technique and interpretation. J Infect Dis 2000;181:S421-5.

11. Terada K, Hiraga Y, Mori R, et al. Double infection of Mycoplasma pneumoniae and Chlamydia pneumoniae in children. Kansenshogaku Zasshi 1996;70:1176-80. 